\title{
Undersampled Radial MRI with Multiple Coils. Iterative Image Reconstruction Using a Total Variation Constraint
}

\author{
Kai Tobias Block,* Martin Uecker, and Jens Frahm
}

\begin{abstract}
The reconstruction of artifact-free images from radially encoded MRI acquisitions poses a difficult task for undersampled data sets, that is for a much lower number of spokes in k-space than data samples per spoke. Here, we developed an iterative reconstruction method for undersampled radial MRI which (i) is based on a nonlinear optimization, (ii) allows for the incorporation of prior knowledge with use of penalty functions, and (iii) deals with data from multiple coils. The procedure arises as a twostep mechanism which first estimates the coil profiles and then renders a final image that complies with the actual observations. Prior knowledge is introduced by penalizing edges in coil profiles and by a total variation constraint for the final image. The latter condition leads to an effective suppression of undersampling (streaking) artifacts and further adds a certain degree of denoising. Apart from simulations, experimental results for a radial spin-echo MRI sequence are presented for phantoms and human brain in vivo at 2.9 T using 24,48 , and 96 spokes with 256 data samples. In comparison to conventional reconstructions (regridding) the proposed method yielded visually improved image quality in all cases. Magn Reson Med 57:1086-1098, 2007. () 2007 Wiley-Liss, Inc.
\end{abstract}

Key words: compressed sensing; inverse problems; iterative reconstruction; projection reconstruction; regridding

\section{INTRODUCTION}

Radial encodings in MRI sample k-space along spokes instead of parallel rows as for conventional phase-encoding schemes. Although radial imaging has already been used by Lauterbur in his seminal paper on MRI (1), pertinent trajectories did not find a wide range of applications. Recently, however, radial acquisition techniques regained strong interest as documented by a number of exciting developments. Prominent examples include highly constrained backprojection approaches for significantly accelerated time-resolved MRI $(2,3)$, k-space weighted projection reconstruction methods for multicontrast MRI $(4,5)$, and techniques for MRI with ultra-short echo times (6).

The renewed interest in radial imaging arises from its unique properties. First of all, each spoke of a radial data set contains an equal amount of low and high spatial frequencies, which leads to advantageous undersampling properties. Second, the Fourier transform of each spoke corresponds to the complex profile of a projection through the object in an angle perpendicular to the direction of the spoke. This relationship is a direct consequence of the Fourier slice theorem and assigns a geometric

Biomedizinische NMR Forschungs $\mathrm{GmbH}$ am Max-Planck-Institut für biophysikalische Chemie, Göttingen, Germany

${ }^{\star}$ Correspondence to: T. Block, Biomedizinische NMR Forschungs $\mathrm{GmbH}$, 37070 Göttingen, Germany. E-mail: tblock@ gwdg.de

Received 30 October 2006; revised 10 January 2007; accepted 21 February 2007.

DOI 10.1002/mrm.21236

Published online in Wiley InterScience (www.interscience.wiley.com).

(C) 2007 Wiley-Liss, Inc. meaning to each single spoke. It allows for the adoption of reconstruction techniques from transmission tomography including consistency criteria, which can be used for artifact correction (7). Third, radial trajectories impose a drastic oversampling of the central portion of k-space which, though apparently inefficient, turns out to be beneficial in certain practical scenarios. For example, it has been consistently reported that radial trajectories provide a low sensitivity to object motion $(8,9)$. Moreover, the central oversampling may be exploited for multicontrast MRI and parallel imaging $(4,10)$ by reconstructing multiple low-resolution images from undersampled data sets.

The advantages of radial imaging are counterbalanced by a number of complications that accompany experimental implementations. First, when using gradient echoes, off-resonance effects from field inhomogeneities may lead to significant image artifacts. Second, due to the nonCartesian sampling of k-space, the image reconstruction process becomes a sophisticated task, which so far hampered the acceptance of radial MRI in many circumstances. While conventional Fourier imaging techniques simply arrange the acquired lines of phase-encoded data on a rectangular grid and perform a $2 \mathrm{D}$ Fourier transformation, radial encodings sample the object's Fourier transform at irregular nonequidistant positions. Thus, it is not intuitively clear how to obtain a rectangular image from the data samples. Moreover, the two commonly used radial image reconstruction schemes, namely filtered back projection and regridding, require the acquisition of a large number of spokes to provide adequate image quality or, conversely, cause substantial image artifacts for undersampled data sets.

The purpose of this work was to design an iterative reconstruction method for undersampled radial MRI, that is for radially encoded MRI data sets with a much lower number of spokes than data samples per spoke. The approach may be exploited to reduce the acquisition time of highresolution images to a degree neither achievable by (partial) Fourier MRI nor conventional reconstructions from projections.

\section{THEORY}

\section{Conventional Radial Image Reconstructions}

MRI acquisitions using radial trajectories are usually reconstructed with either projection reconstruction or regridding methods. Projection reconstruction is based on the Fourier slice theorem, which allows to recast the problem into an image reconstruction from projection profiles, that is the Fourier transforms of the acquired spokes. The filtered backprojection method, originally developed for X-ray computed tomography, can then be applied to obtain the image by smearing all projection profiles over a matrix in a direction opposite to that of each profile (11). Prior to 
this backprojection step it is necessary to compensate for the oversampling of the k-space center which is usually accomplished by filtering the profiles with the well-known ramp filter $|k|$.

The more frequently used regridding technique interpolates the data onto a rectangular grid in the frequency domain and subsequently performs a Fourier transformation (12). The interpolation is done by convolving the spoke data with an approximate sinc kernel followed by an evaluation of the convolved data at the grid positions. The Kaiser-Bessel window has been shown to provide a good interpolation quality at a reasonable window width. It is typically used as interpolation kernel (13)

$$
C_{\mathrm{KB}}(k)=\left\{\begin{array}{ll}
\frac{1}{L} I_{0}\left(\beta \sqrt{1-(2 k / L)^{2}}\right) & |k| \leq \frac{L}{2} \\
0 & |k|>\frac{L}{2}
\end{array},\right.
$$

with $L$ the desired kernel width, $I_{0}(k)$ the zero-order modified Bessel function of the first kind, and $\beta$ a shape parameter which can be selected according to an equation reported by Beatty et al. (14). The convolution with the Kaiser-Bessel window in the frequency domain leads to an undesired intensity modulation in the image domain. This so-called roll-off effect can be compensated for by dividing the image by the (approximate) Fourier transform of the Kaiser-Bessel window right after Fourier transformation

$$
C_{\mathrm{KB}}(x)=\frac{\sin \sqrt{(\pi L x)^{2}-\beta^{2}}}{\sqrt{(\pi L x)^{2}-\beta^{2}}} .
$$

Similar to projection reconstruction, it is necessary to compensate for the varying sample density of the trajectory which again can be achieved by weighting the data with the ramp filter $|k|$ before performing the convolution. In the context of regridding, this strategy is termed density compensation.

Basically, radial image reconstruction by projection reconstruction and regridding is equivalent. The major difference between both approaches is the (frequency or image) domain where the interpolation step is carried out. Indeed, both approaches yield very similar results which only differ with respect to the applied interpolation. Therefore, we limit the following discussion to the regridding approach.

For a sufficiently high number of spokes, regridding allows for an accurate reconstruction of the object as shown in Fig. 1 for the case of 402 spokes (256 sample points each). In fact, according to the literature, $\frac{\pi}{2} \cdot n$ spokes have to be obtained for an image with a base resolution of $n \times n$ sample points (15). While this criterion ensures that the outmost samples of two neighbouring spokes have a maximum distance of $\Delta k=\frac{1}{\text { FOV }}$ in line with the well-known Nyquist condition for conventional Fourier imaging, it prolongs the acquisition of a radial image by about $57 \%$ relative to that of a corresponding fully sampled Fourier image.

If one reduces the number of acquired spokes far below the recommended value, the reconstructed image presents with two characteristic features: while most object features remain visible at good spatial resolution, the use of a regridding (or filtered back projection) algorithm results

FIG. 1. Regridding reconstructions (Shepp-Logan phantom, $256 \times 256$ matrix) using simulated data from 402, 64, and 24 spokes (256 data samples). The lower right panel shows the Fourier transform of the image reconstructed from 24 spokes. It reveals unmeasured gaps in k-space in between spokes (arrows). The reconstructions from 64 and 24 spokes suffer from streaking artifacts caused by undersampling.
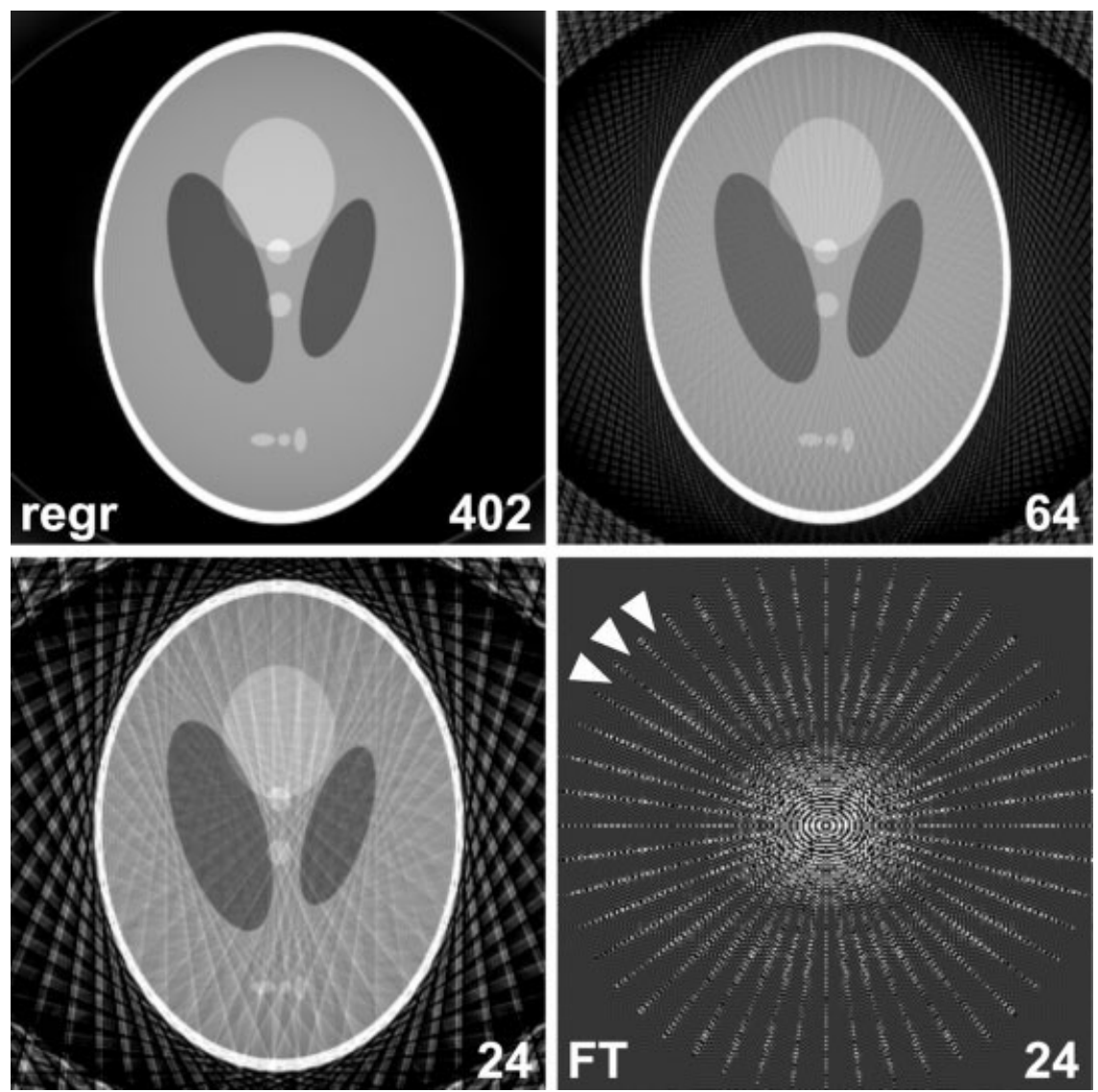
in so-called streaking artifacts. Both properties are demonstrated in Fig. 1 for a reduction of the number of spokes from 402 to 64 or even 24. Although the strength of the streaking artifacts increases with the extent of undersampling, it is remarkable how much information about the object can still be seen in an undersampled image with only 24 spokes. The origin of the streaking artifacts may be best understood when considering the Fourier transform of the undersampled image also shown in Fig. 1. The resulting k-space pattern matches the acquired data at the spoke positions, but in-between the Fourier transform is zero (except for a small surrounding of the spokes resulting from the convolution). Obviously, this solution of piecewise constant areas and many edges is not an accurate representation of the Fourier transform of the true image which explains the failure of the regridding method.

\section{Radial Image Reconstruction as Inverse Problem}

Suppose we stack the measured data from all spokes into a vector $\vec{y}$, where each entry $y_{i}$ corresponds to a single sample, then the image reconstruction process can be seen as estimating a stacked image vector $\vec{x}$ with $n \times n$ pixel intensities $x_{i}$ from the given data vector $\vec{y}$. When acquiring only a limited number of spokes, the size of the data vector $\vec{y}$ is usually smaller than the desired image vector $\vec{x}$. Because in this case the problem is underdetermined, one may address it in the opposite direction: suppose we have given an image $\vec{X}$ and want to calculate the corresponding data vector $\vec{y}$. This can be achieved by a Fourier transformation of the image and an evaluation of the image's Fourier transform at the trajectory positions using a k-space interpolation. The necessary linear operations can be combined into a single matrix $\mathcal{A}$, denoted as system matrix, so that the forward problem may be written as

$$
\vec{y}=\mathcal{A} \vec{x} .
$$

Instead of trying to directly invert this equation to obtain an image vector $\vec{x}$ from a given data vector $\vec{y}$, it is more advantageous to iteratively estimate an image vector $\vec{x}$ that fits to the given data vector $\vec{y}$. This is because the problem is not only ill-posed but also very large.

How well the image estimate fits to the measured data can be measured by calculating the $L_{2}$ norm of the residuum

$$
\Phi(\vec{x})=\frac{1}{2}\|\mathcal{A} \vec{x}-\vec{y}\|_{2}^{2}
$$

Because we want to find an image that best represents the measured data, we are looking for a vector $\vec{x}$ that minimizes the functional [4]

$$
\vec{X}=\underset{\vec{x}}{\operatorname{argmin}} \Phi(\vec{x})
$$

Finding a solution to this equation requires a highly efficient optimization method due to the large size of the parameter space. A suitable approach for such problems is the conjugate gradient method. It has initially been presented by Hestenes and Stiefel in 1952 for the solution of linear systems and in the meantime successfully applied to MRI reconstruction problems (16). The method has been extended to nonlinear optimization by Fletcher and Reeves in 1964 and since then a number of optimized nonlinear conjugate gradient approaches have been developed (17). Recently, Hager and Zhang (18) presented a version with improved convergence properties, which we found appropriate to solve Eq. [5].

The conjugate gradient method is an iterative two-step scheme, which is repeated until a satisfying solution has been found. First, a search direction is estimated in parameter space and, second, a line search into that direction is performed until the minimum of the functional in this direction has been identified. The search direction is obtained by calculating the gradient at the actual estimate and by superposing it with the prior search direction scaled by a factor that guarantees the conjugacy of successive search directions. The gradient of the functional [4] is given by

$$
\nabla \Phi(\vec{x})=\mathcal{A}^{\sharp} \mathcal{A} \vec{x}-\mathcal{A}^{\sharp} \vec{y}=\mathcal{A}^{\sharp}(\mathcal{A} \vec{x}-\vec{y}),
$$

where $\mathcal{A}^{\sharp}$ denotes the adjoint matrix to $\mathcal{A}$, that is the transposed matrix with each entry replaced by its complex conjugate. As the matrix $\mathcal{A}$ performs a Fourier transformation followed by an interpolation to the spokes, the matrix $\mathcal{A}^{\sharp}$ performs an interpolation from the spokes to a grid followed by an inverse Fourier transformation. It is important to point out that $\mathcal{A}^{\sharp}$ is not the inverse matrix to $\mathcal{A}$. Obviously, the optimum image estimate is reached if the gradient of the functional vanishes.

The right part of Eq. [6] gives insight into how the reconstruction process works. At every step of the algorithm, the actual image estimate $\vec{X}$ is mapped to the frequency domain by multiplication with $\mathcal{A}$. It is then compared how well the estimate fits to the measured data by calculating the difference to $\vec{y}$. If the estimate is good enough, then the residuum vector contains only small entries, otherwise it contains large entries. In this case, the algorithm needs to know how to modify the image estimate in order to improve the match of the samples in the frequency domain. This information is obtained by mapping the residuum back to the image space by matrix multiplication with $\mathcal{A}^{\sharp}$.

The middle part of Eq. [6] allows for another view of the reconstruction process. If the trajectory undersamples k-space, information is lost when applying matrix $\mathcal{A}$ to obtain the spoke data that corresponds to the image estimate. This can be seen as projecting the image's Fourier transform to the spokes of the trajectory. Successive application of $\mathcal{A}^{\sharp}$, that is $\mathcal{A}^{\sharp} \mathcal{A} \vec{x}$, may then be understood as convolving or blurring the actual image estimate with the point spread function of the trajectory. A multiplication of the adjoint matrix $\mathcal{A}^{\sharp}$ with the data vector $\vec{y}$ gives an image comparable to that of a regridding solution (except for the missing density compensation). Trying to match the blurred image estimate with the quasi regridding image, which is also blurred due to undersampling, is a general deconvolution approach. This has previously been pointed out by Delaney and Bresler (19) for the case of iterative parallel-beam tomography reconstruction. Indeed, Eq. [4] has the same form as common approaches used in image restoration and image denoising. The quality of the deblurring depends on how well the system matrix $\mathcal{A}$ models the true process underlying the generation of the data vector $\vec{y}$. 

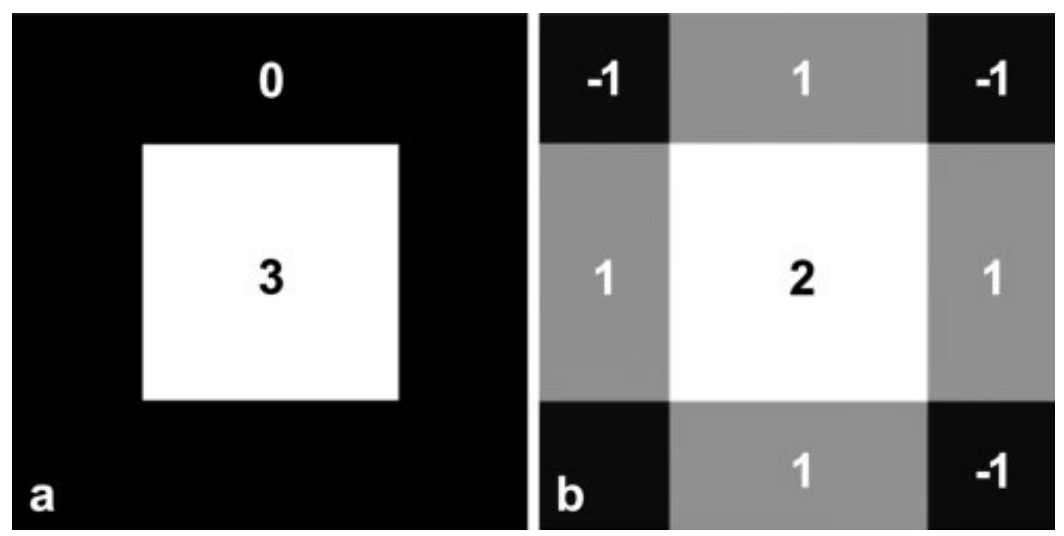

FIG. 2. Illustration of two equally valid reconstructions of a rectangle from only two radial projections. (a) True solution and (b) solution suffering from streaking artifacts. Both solutions are identical at the measured positions (spokes) in k-space. Numbers indicate hypothetical pixel intensities.

\section{Regularization by A Priori Information}

The reconstruction of an undersampled radial image by optimizing Eq. [4] still leads to streaking artifacts. This is not surprising as the procedure does not measure the accuracy of the estimate at any other position in k-space than at the positions of the spokes. For illustration, Fig. 2 shows two reconstructions of a rectangle from just two spokes. While Fig. 2a represents the true object, the image in Fig. 2b is degraded by streaking artifacts. Equation [4] cannot tell which solution is better, because both solutions yield an identical pattern in k-space at the positions of the spokes. Differences only occur in between spokes. To obtain a better estimate than the regridding solution, it is therefore necessary to extend the functional [4] by criteria that introduce some kind of quality weighting by adding penalties. This concept is referred to as regularization and, of course, requires some a priori knowledge about the true object. The challenge in selecting respective criteria is that they should not be too specific about the object and keep the problem optimizable. This requires the penalties to be convex functions, which allow for global optimization. Accordingly, the regularized functional takes the form

$$
\Phi(\vec{x})=\frac{1}{2}\|\mathcal{A} \vec{x}-\vec{y}\|_{2}^{2}+\sum_{i} \lambda_{i} R_{i}(\vec{x}),
$$

where $R_{i}(\vec{x})$ are the penalty functions. The coefficients $\lambda_{i}$ represent tuning factors that allow for shifting the preference from matching the image to the measured data to satisfying the a priori knowledge. In fact, because measured data is contaminated by noise, the search for a perfect match of the image estimate to the measured data is usually not a good strategy as is it drives the image estimate to render the experimental noise. To compensate for this effect, it is necessary to adjust the coefficients $\lambda_{i}$ in accordance with the signal-to-noise ratio of the acquired data.

For radial MRI, there are several choices of how to restrict the solution space of the image estimation process. If knowledge about the size of the object is available, it is possible to penalize image intensity outside of the potential object. In particular, due to the rotational symmetry of radial sampling, all image intensity outside a circular field of view (FOV) can be usually considered as artifactual. A corresponding penalty function can be formulated as

$$
R_{\mathrm{FOV}}(\vec{x})=\sum_{i} \phi\left(x_{i}\right)
$$

where

$$
\phi\left(x_{i}\right)= \begin{cases}\left|x_{i}\right|^{2} & x_{i} \notin \mathrm{cFOV} \\ 0 & x_{i} \in \mathrm{cFOV}\end{cases}
$$

and cFOV denotes the circular FOV.

Another penalty, which turned out to be very effective in general image restoration, is the restriction of parameter space to positive values. It can be achieved by using the penalty function

$$
R_{\mathrm{pos}}(\vec{x})=\sum_{i} \varphi\left(x_{i}\right)
$$

where

$$
\varphi\left(x_{i}\right)=\left\{\begin{array}{ll}
x_{i}^{2} & x_{i}<0 \\
0 & x_{i} \geq 0
\end{array} .\right.
$$

Suppression of negative values prevents the algorithm from inserting negative fill values into the image, a tendency often performed by the unconstrained algorithm to better match the measured data. This, however, leads to inaccurate image estimates.

At first glance, the exclusion of negative values seems to ideally apply to the MRI situation where the measured physical quantity, that is the proton density modulated by some relaxation process, is a positive unit. Unfortunately, however, the use of phased array coils with a complex sensitivity profile as well as the occurrence of phase variations within the object forbid the direct application of this criterion. In most imaging situations, neither the real nor the imaginary part of the image can be restricted to positive values.

A third penalty, which has been successfully used in image restoration, is the restriction of total variation (TV) initially presented by Rudin et al. in 1992 for noise removal (20). The basic assumption of this idea is that the object consists of areas with constant (or only mildly varying) intensity, which applies quite well to medical tomographic images. If the object is piecewise constant, then the best representation of all image estimates that match at the spoke positions should be given by the one with the lowest 

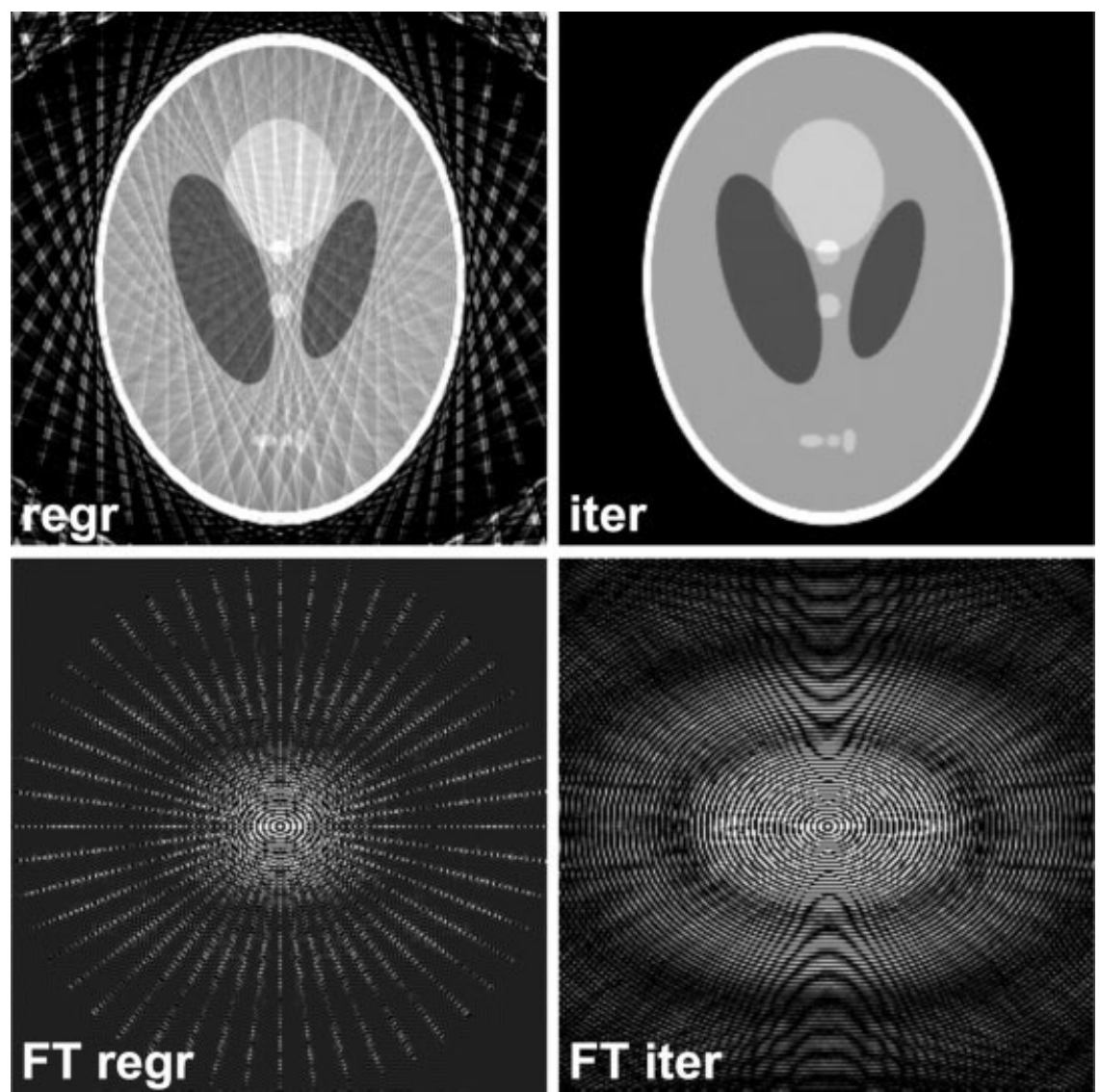

FIG. 3. Radial image reconstructions (Shepp-Logan phantom, $256 \times 256$ matrix), using simulated data from 24 spokes (256 samples). (Top left) Regridding and (top right) the proposed iterative technique with prior knowledge. (Bottom) Corresponding Fourier transforms. The iterative technique reconstructs the image of the object without streaking artifacts. Accordingly, its Fourier transform recovers the unmeasured gaps in k-space in-between spokes. derivatives at all pixel positions, that is the one minimizing the total variation

$$
R_{\mathrm{TV}}(\vec{x})=\sum_{i}\left|D_{x}\left(x_{i}\right)\right|+\left|D_{y}\left(x_{i}\right)\right|,
$$

where $D_{x}$ and $D_{y}$ denote the derivatives in $x$ and $y$ direction, repectively. The first order derivative at the pixel position $(n, m)$ can be calculated from the finite difference between neighboring pixels

$$
\begin{aligned}
& D_{x}^{(1)}(m, n)=x(m, n)-x(m-1, n) \\
& D_{y}^{(1)}(m, n)=x(m, n)-x(m, n-1) .
\end{aligned}
$$

It is important to note that the total variation in Eq. [12] depends on the modulus of the derivatives. This dependency, well-known in the context of $L_{1}$ optimization, ensures edge preservation in the image and especially penalizes oscillations, which helps to suppress Gibbs ringing artifacts as well as noise. Replacing the modulus by a square dependency leads to an image with global smoothness because intensity changes between neighboring pixels become very strongly penalized.

The simple use of first order derivatives for the total variation constraint [12] may create patchy images as it tends to generate regions with constant intensity. It is therefore preferable to rely on the second order derivatives, which then allows for image regions with constant intensity gradients

$$
\begin{array}{r}
D_{x}^{(2)}(m, n)=x(m-1, n)-2 \cdot x(m, n)+x(m+1, n) \\
D_{y}^{(2)}(m, n)=x(m, n-1)-2 \cdot x(m, n)+x(m, n+1) \\
D_{x y}^{(2)}(m, n)=x(m, n)-x(m-1, n) \\
\quad-x(m, n-1)+x(m-1, n-1) .
\end{array}
$$

As pointed out by Geman and Yang (21), it is sometimes advantageous to use a combination of first and second order derivatives.

The upper row of Fig. 3 shows reconstructions of the Shepp-Logan phantom from 24 spokes obtained either by regridding or the proposed inverse formulation with penalties as presented in this section. A comparison of the images clearly demonstrates the superior performance of the new method in reducing streaking artifacts which for the simulated data have been effectively removed. The lower row of Fig. 3 depicts the corresponding Fourier transforms. It turns out that the incorporation of a priori information by appropriate penalty functions leads to a proper recovery of k-space representations in between the spokes. Figure 4 shows a schematic flow chart of the iterative reconstruction technique.

\section{Radial Image Reconstruction for Multiple Coils}

Two more difficulties arise when employing the described iterative strategy for the reconstruction of real MRI data. 
FIG. 4. Schematic diagram of the proposed iterative reconstruction technique. The procedure has been formulated as an inverse problem. The solution employs a nonlinear conjugate gradient method to obtain an image estimate that complies with the measured data as well as prior knowledge.

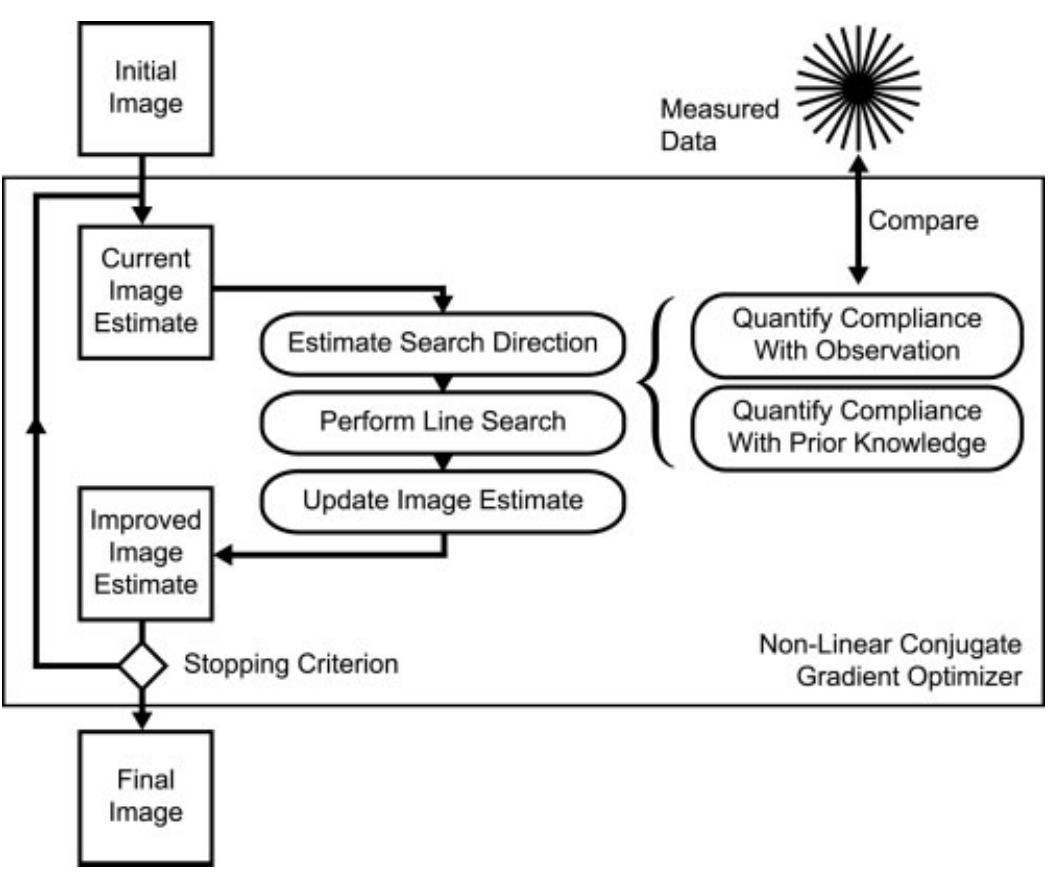

First, the acquired signal is complex and, although the final result is a real valued image, it is only possible to remove the phase after spatially resolving the object. While this is usually done by calculating the magnitude, this operation is not linear and cannot be integrated into the system matrix, so that it remains necessary to deal with the complex nature of the images. Second, modern MRI systems use phased-array coils each of which has a different intensity profile and a variable phase. Again, a suitable combination of the signals from all individual coils can only be obtained after spatially resolving the object. As a consequence, any radial image reconstruction has to cope with the different coil signals during the entire process.

To this end we propose an iterative two-step reconstruction approach. The first step attempts to obtain profiles for all coils, which are then used in the iterations of the second step to combine the single coil channels and to remove the phase each time when mapping between the frequency domain and image space. Thus, the second step renders a combined and real valued image. An attractive feature of this approach is that no reference data nor shared data is needed to estimate the coil profiles.

In the first step, the signals from all coil channels are handled separately. The real and imaginary parts are treated as independent parameters leading to a complex valued image estimate for every coil channel. It is known that MRI coil profiles are smooth functions that vary only slowly and do not have sharp edges. This knowledge is incorporated by using quadratic regularization of the image derivatives, which leads to globally smooth images as pointed out before

$$
R_{\text {coil }}(\vec{x})=\sum_{i} D_{x}\left(x_{i}\right)^{2}+D_{y}\left(x_{i}\right)^{2},
$$

where $D_{x}$ and $D_{y}$ are the known derivative operators. After finishing the iterations for all coils, a sum-of-squares image is created. A division of the single channel images by the sum-of-squares image yields the respective coil profiles. Noteworthy, these estimated coil profiles also include the phase variations related to the object as the real valued sumof-squares image has been taken as a reference. Because the penalty function $R_{\text {coil }}(\vec{x})$ depends quadratically on $\vec{x}$, the line search that is part of the conjugate gradient iteration, requires only one step and only a low number of iterations is needed to obtain a reasonable image. Therefore, the coil profile estimation step takes only moderate computational time.

For the second reconstruction step, the raw data from all coil channels is stacked into the data vector $\vec{y}$. The system matrix $\mathcal{A}$ is extended by a multiplication with the corresponding coil profile before performing the Fourier transformation for every channel. Figure 5 shows a diagram of the operations that are executed by the system matrix $\mathcal{A}$ and the adjoint matrix $\mathcal{A}^{\sharp}$ to map between frequency and image space. By combining data available from all coil channels into the data vector $\vec{y}$, the algorithm renders an image estimate that complies with the observations from all coils. Further, removing the phase variations using the estimated coil profiles allows to discard the imaginary part of the image estimates as well as to apply constraints based on non-negativity, which otherwise would not be possible. Noteworthy, a combined coil reconstruction ensures that the total variation constraint remains applicable in a phased-array setup. Otherwise, the intensity modulation of the coil profiles would conflict with the idea of piecewise constant images.

\section{MATERIALS AND METHODS}

\section{MRI Data Acquisition}

As a first proof-of-principle application of the proposed technique for reconstructing undersampled radial images, we acquired data with a radial 2D spin-echo MRI sequence. Spin echoes rather than gradient echoes were chosen 
System matrix A

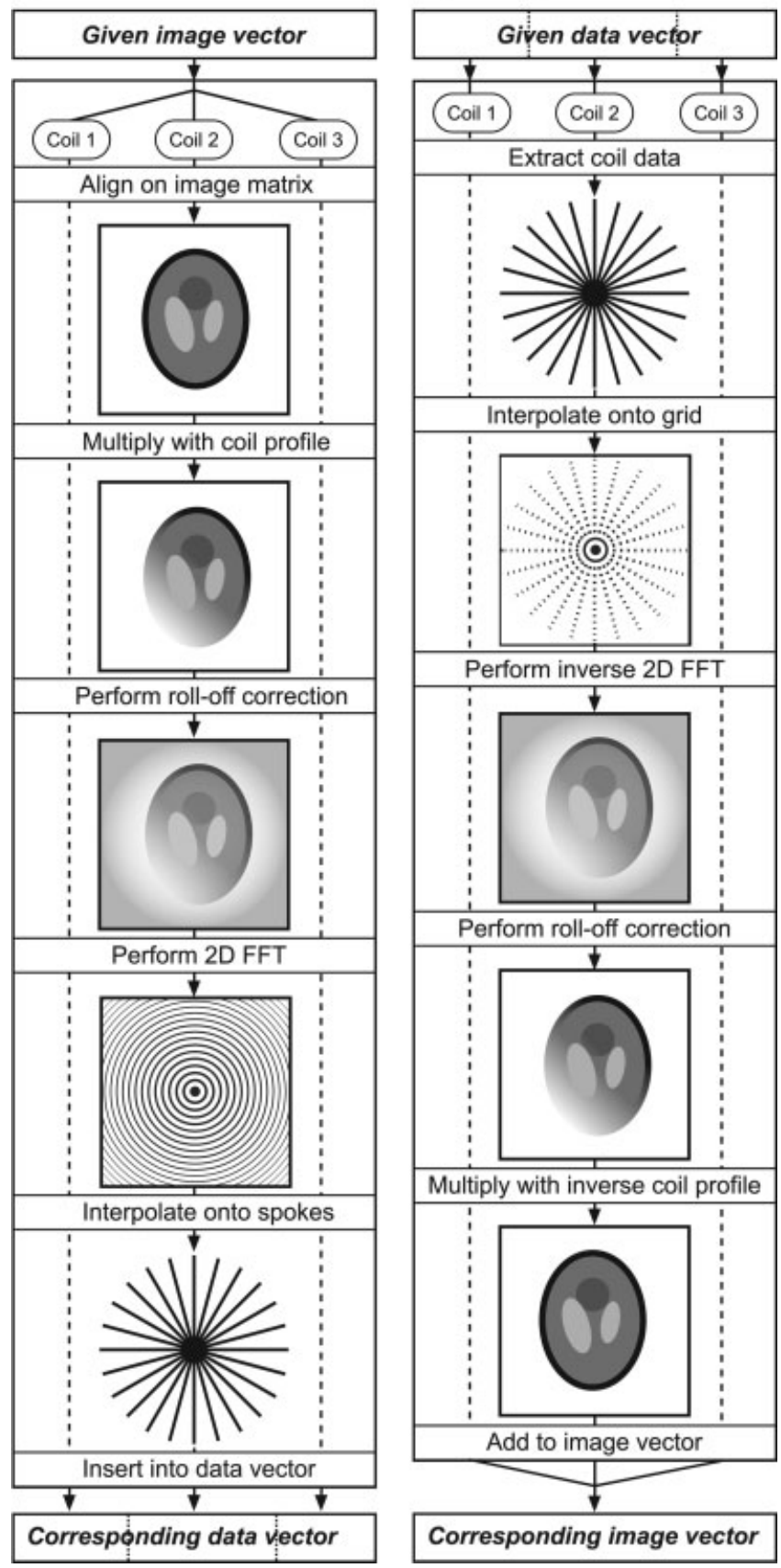

FIG. 5. Schematic diagram of the procedural implementations of (left) the system matrix $\mathcal{A}$ and (right) the adjoint system matrix $\mathcal{A}^{\sharp}$ that are used to map from image domain to frequency domain and vice versa. For details see text.

to avoid putative complications from the sensitivity to off-resonance effects, which are not related to the reconstruction process studied here. All measurements were conducted at 2.9 T (Siemens Magnetom TIM Trio, Erlangen, Germany) using a receive-only 12-channel head coil in triple mode yielding 12 channels with different combinations of the coils. Written informed consent was obtained from all subjects prior to the examination.
The MRI sequence was derived from a standard spinecho 2D sequence of the manufacturer and modified to radial acquisitions with a readout oversampling factor of two. As radial imaging is sensitive to gradient timing errors, we used a technique presented by Speier and Trautwein (22) to correct for such errors. In fact, gradient deviations cause an incorrect trajectory and lead to smearing artifacts in a regridding reconstruction that may become emphasized by iterative reconstructions due to repeated imprecise interpolations. After calibration of the MRI system the chosen correction effectively suppressed respective artifacts.

All images were acquired with a base resolution of 256 pixels covering a $230 \mathrm{~mm}$ FOV (slice thickness $2 \mathrm{~mm}$ ). The number of spokes varied from 8 to 96 . The phantom images were acquired with a repetition time TR $=4000 \mathrm{~ms}$ and echo time $\mathrm{TE}=11 \mathrm{~ms}$ (bandwidth $180 \mathrm{~Hz} /$ pixel), while the in vivo images of the human brain were acquired with $\mathrm{TR} / \mathrm{TE}=2500 / 50 \mathrm{~ms}$ (bandwidth $180 \mathrm{~Hz} /$ pixel) and $\mathrm{TR} / \mathrm{TE}=3000 / 80 \mathrm{~ms}$ (bandwidth $90 \mathrm{~Hz} /$ pixel) for $\mathrm{T} 2$ contrast.

\section{Radial Image Reconstruction}

Our current implementation involves an online regridding reconstruction of radial images. Subsequently, the acquired raw data is exported from the scanner and reconstructed offline using our in-house software package MRISim, which has been written in $\mathrm{C} / \mathrm{C}++$ using the libraries GNU Scientific Library, FFTW3, QT4, Blitz++, and the nonlinear solver bench from the restoreInpaint project.

A look-up table is calculated in a preparation step to speed up the interpolation operations that are repeatedly carried out within the iterations. The look-up table contains all coefficients needed to interpolate from spoke to grid data and vice versa. The coefficients are calculated using a Kaiser-Bessel window given by Eq. [1] with $L=6$ and $\beta=13.8551$. Further, a matrix containing the values for the roll-off correction is precalculated.

Prior to starting the iterations for a particular image, the phase offset of the central k-space sample of each spoke is determined and removed from the spoke data. This step corrects for interference artifacts that arise due to the overlapping k-space coverage of the spokes when a phase offset between single spokes is present. Such phase offset deviations are, for example, caused by through-plane motion.

A second preprocessing step exploits the fact that the zeroth moment (or sum) of a projection through an object is independent of the projection angle (7). Thus, the zeroth moment of the projections can be used to perform a firstorder correction for spoke intensity deviations which, for instance, can occur when measuring in a transient phase of the magnetization, that is during the approach to steadystate conditions. In more detail, the data of each spoke is Fourier transformed, the zeroth moment is calculated, and the resulting spoke intensity is used in the iterations to weight the calculated spokes before matching them to the measured data. The procedure eliminates potential smearing artifacts. 
In the coil estimation step, we use a moderate penalty for image intensity outside the cFOV by setting $\lambda_{\text {FOV }}=1$ in Eq. [7]. Edges are strongly penalized by quadratically constraining the first-order derivatives of the image intensity using Eq. [15] with $\lambda_{\text {coil }}=10$. In the final image reconstruction step, we strongly penalized image intensity outside the cFOV by setting a high value for the corresponding coefficient $\lambda_{\mathrm{FOV}}$ or even reject all intensity in this area. To incorporate the total variation constraint, the magnitude of the image derivatives was penalized with a weighting of 0.77 for the first-order and 0.23 for the second-order as suggested by Geman and Yang (21). A value of $\lambda_{\mathrm{TV}}=0.0001$ for the total variation penalty turned out as a robust choice for our present study. Further, we penalized negative values by setting $\lambda_{\text {pos }}=5$, which stabilized the convergence process but also slowed it down.

Currently, we are running the reconstruction steps for a fixed number of iterations. The images presented in this work were rendered using 30 iterations for the coil estimation and 120 iterations for the final image reconstruction step. However, in many cases, a reasonable image quality was also obtained with a much smaller number of iterations, typical numbers being 10 iterations for the coil estimation and 20 iterations for the final reconstruction step.

\section{RESULTS}

\section{Phantom Studies}

Figure 6 shows experimental coil profiles that were estimated by the coil estimation step from a data set of 48 spokes obtained for a phantom. The profiles are smooth inside the object and do not contain visible object features as expected. The algorithm is unable to determine the coil profile outside the object due to the absence of any signal, but this poses no problem for the image reconstruction. The corresponding images of the phantom are summarized in Fig. 7 together with reconstructions for 96 and only 24 spokes. For comparison, the upper row shows the results of the regridding approach with a sum-of-squares combination of the multiple coil images.

Obviously, regridding reconstructions suffer from streaking artifacts that increase with decreasing number of spokes. In contrast, the iterative approach renders images without any visible or at least strongly reduced streaking artifacts while maintaining sharp edges in line with findings by Chang et al. using a related approach (23). The proposed method is able to reconstruct a high-quality image of the object from only 48 spokes. In fact, there is only a slight gain in image quality when the number of spokes is increased. In the case of 24 spokes, the algorithm again outperforms the regridding solution. Nevertheless, the method fails in fully recovering the true object and residual streaking artifacts remain visible. However, it should be noted that a reconstruction from 24 spokes corresponds to a data reduction factor of more than 16 compared to the 402 spokes recommended for a $256 \times 256$ image.

\section{In Vivo Studies}

Similar to the phantom studies, Fig. 8 compares reconstructions of radial images from the human brain in vivo using regridding and the proposed iterative method for 96, 48, and 24 spokes. Again, regridding suffers from pronounced streaking artifacts outside as well as inside of the
FIG. 6. Estimated MRI coil profiles (phantom, $256 \times 256$ matrix), using experimental data from 48 spokes (256 samples) and the proposed iterative technique. The profiles correspond to the four primary modes of the 12-channel receive-only head coil. The MRI sequence was a radial spin-echo sequence (TR/TE $=4000 / 11 \mathrm{~ms}, 230 \mathrm{~mm}$ FOV, bandwidth $180 \mathrm{~Hz} /$ pixel).

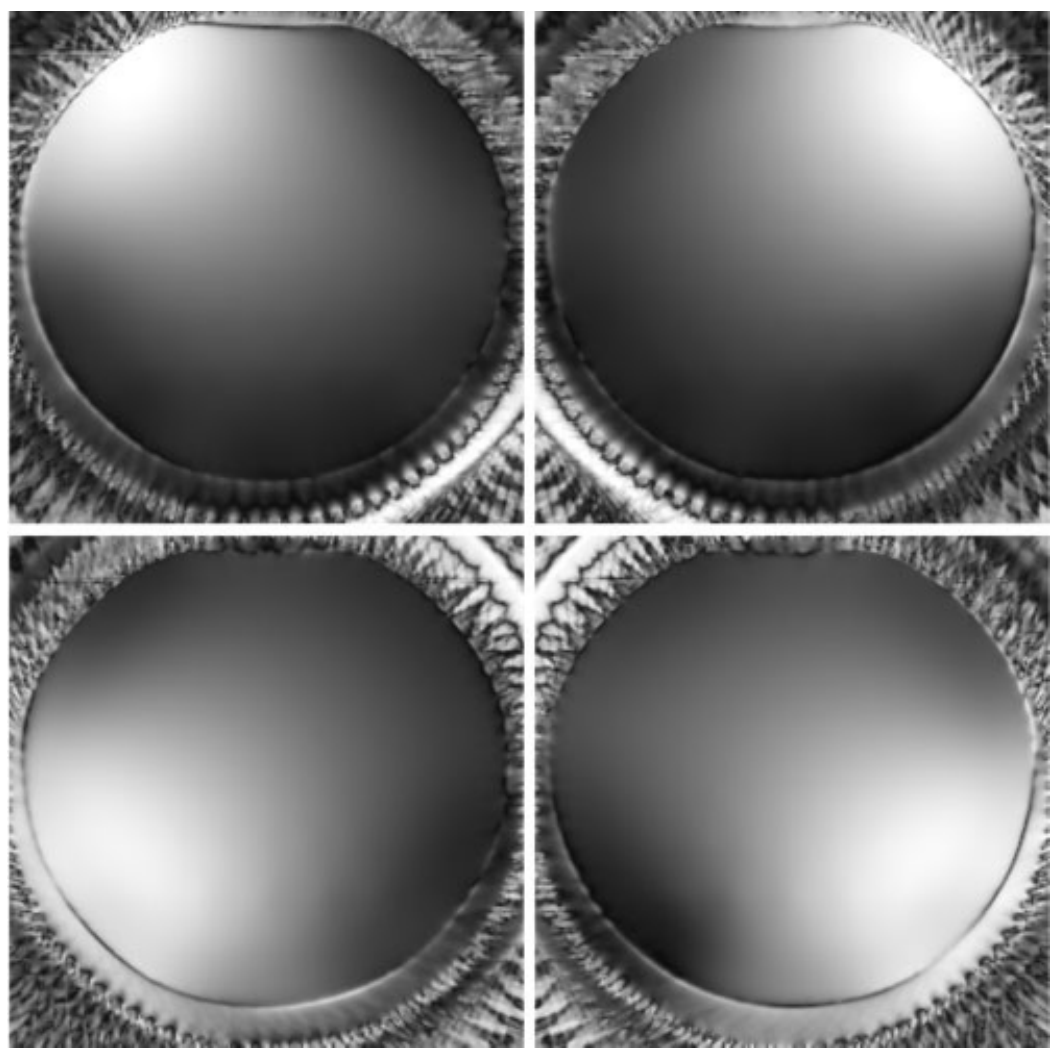


FIG. 7. Radial image reconstructions (phantom, $256 \times 256$ matrix), using experimental data from 96, 48, and 24 spokes (256 samples). (Top) Regridding and (bottom) the proposed iterative technique. Parameters as in Fig. 6.
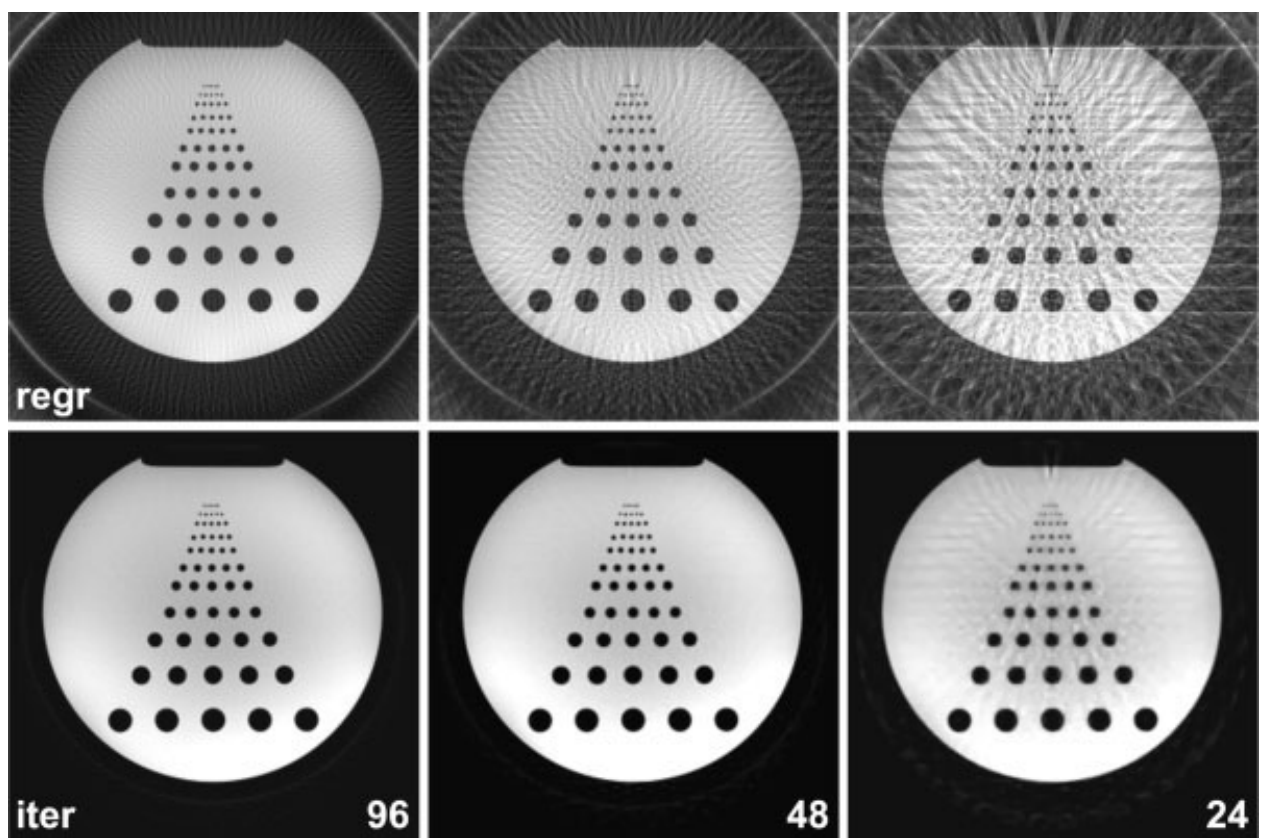

brain, although the artifacts are less clearly visible than in the phantom images due to the occurrence of more complex structural details. Conversely, streaking artifacts are removed (48 spokes) or at least noticeably reduced (24 spokes) when using the iterative approach. Further, the application of the total variation penalty leads to a marked denoising of the images.

The improvement in image quality of the iterative reconstruction technique relative to regridding is even more visible in Fig. 9 magnifying parts of the brain sections from Fig. 8 by a factor of three. To demonstrate the limits, Fig. 10 compares brain sections obtained by iterative reconstructions from 48, 32, 24, 16, 12, and 8 spokes. Of course, the data reduction from 48 to 8 spokes is accompanied by a loss of resolution. The effect is best appreciated for selected fine structures, whereas gross anatomical features such as the ventricles are less affected. Simultaneously, total variation ensures a pronounced reduction of the noise, so that the overall image appearance even for extreme undersampling is of surprising quality.

\section{DISCUSSION}

Remaining Artifacts

The results shown in Figs. 7-10 demonstrate that the proposed reconstruction technique for undersampled radial
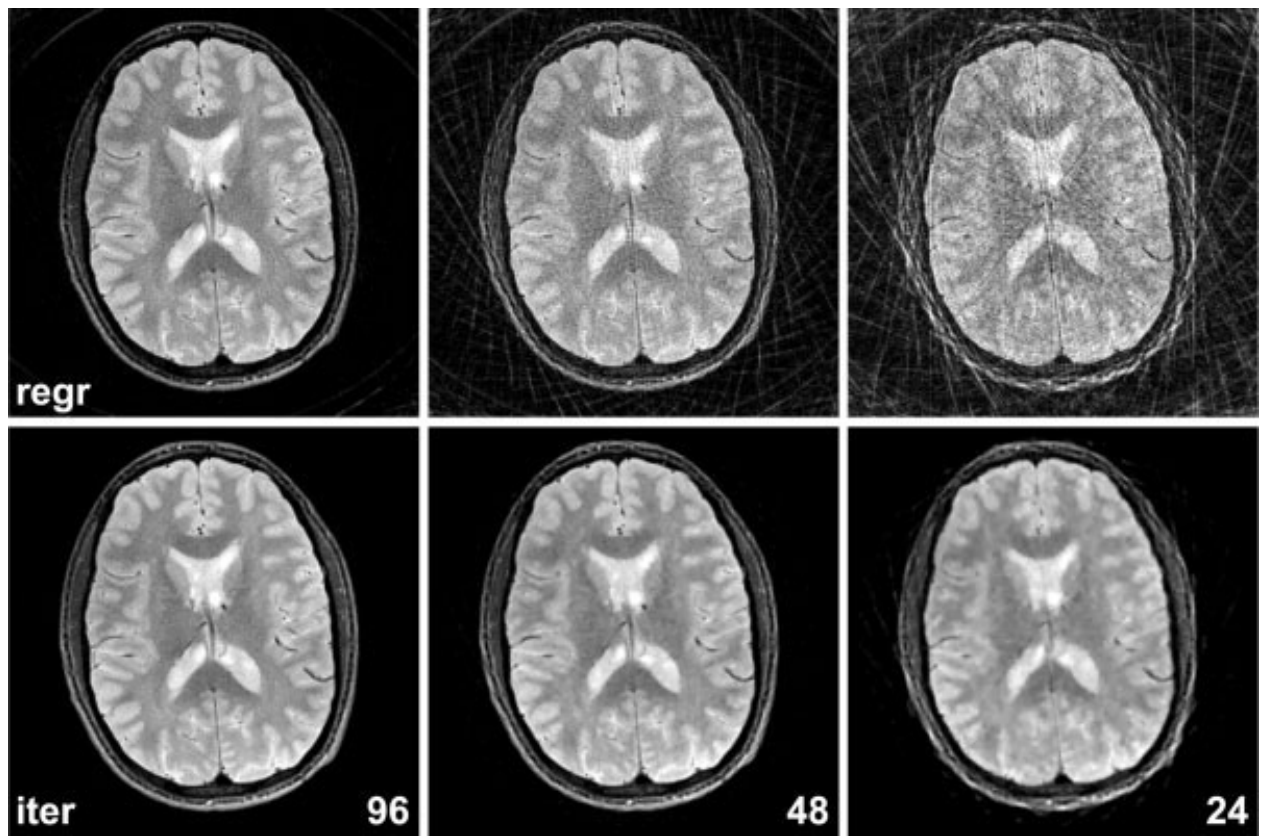

FIG. 8. Radial image reconstructions (human brain, $256 \times 256$ matrix), using experimental data from 96, 48, and 24 spokes (256 samples). (Top) Regridding and (bottom) the proposed iterative technique. Parameters as in Fig. 6 except for $\mathrm{TR} / \mathrm{TE}=2500 / 50 \mathrm{~ms}$. 

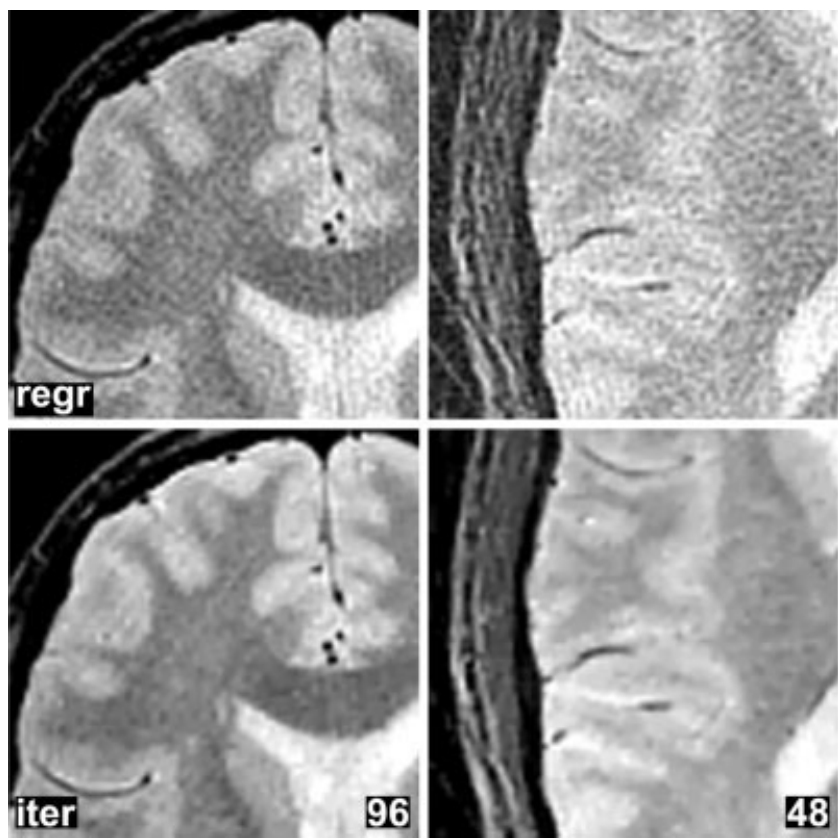

96

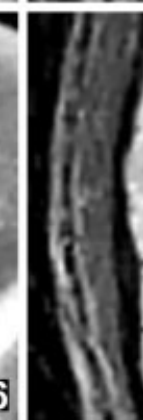

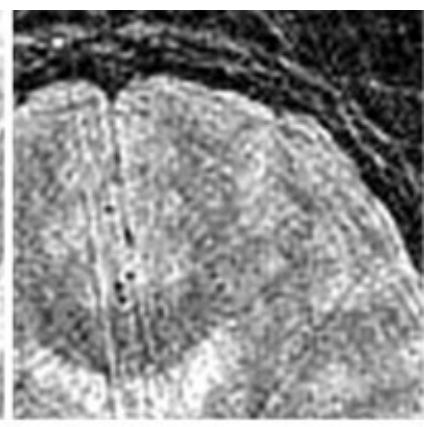

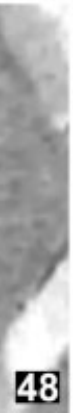

48

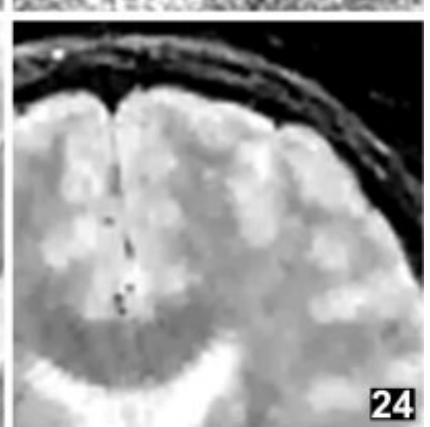

FIG. 9. Magnified views of the same data as shown in Fig. 8.
MRI yields images with clearly improved quality over the conventional regridding approach. Nevertheless, the algorithm is not able to fully remove the streaking artifacts in the heavily undersampled case of 24 spokes. This effect can be explained by closer inspection of the total variation constraint, which plays a central role for the removal of such artifacts or, respectively, the recovery of the interspoke $\mathrm{k}$-space information in the frequency domain.

The total variation concept is based on the assumption that the object is piecewise constant, which implies that only a limited number of edges and intensity jumps are present in the image. According to the underlying theory of compressed sensing, it is under certain circumstances possible to recover a signal from undersampled data, if a basis exists in which the signal can be represented sparsely (24). For total variation, this basis is given by the image's derivative. Thus, the object can be recovered, if it can be represented by a limited or sparse number of edges. This condition is obviously fulfilled by the Shepp-Logan phantom shown in Fig. 3. In this case, the major contribution to the total variation of the regridding solution comes from the streaks that overlap to form a texture-like pattern in the image. These artifacts can be removed by minimizing the total variation, so that the object can be perfectly recovered from only 24 spokes-also stated by Candes et al. (25). However, the experimental data presented in Figs. 7-10 fulfills the needed condition less optimal as the object itself contributes to the total variation of the image, or
FIG. 10. Radial image reconstructions (human brain, $256 \times$ 256 matrix), using experimental data from $48,32,24,16$, 12 , and 8 spokes (256 samples) using the proposed iterative technique. The MRI sequence was a radial spin-echo sequence with fat suppression (TR/TE = $3000 / 80 \mathrm{~ms}, 230 \mathrm{~mm}$ FOV, bandwidth $90 \mathrm{~Hz} /$ pixel).
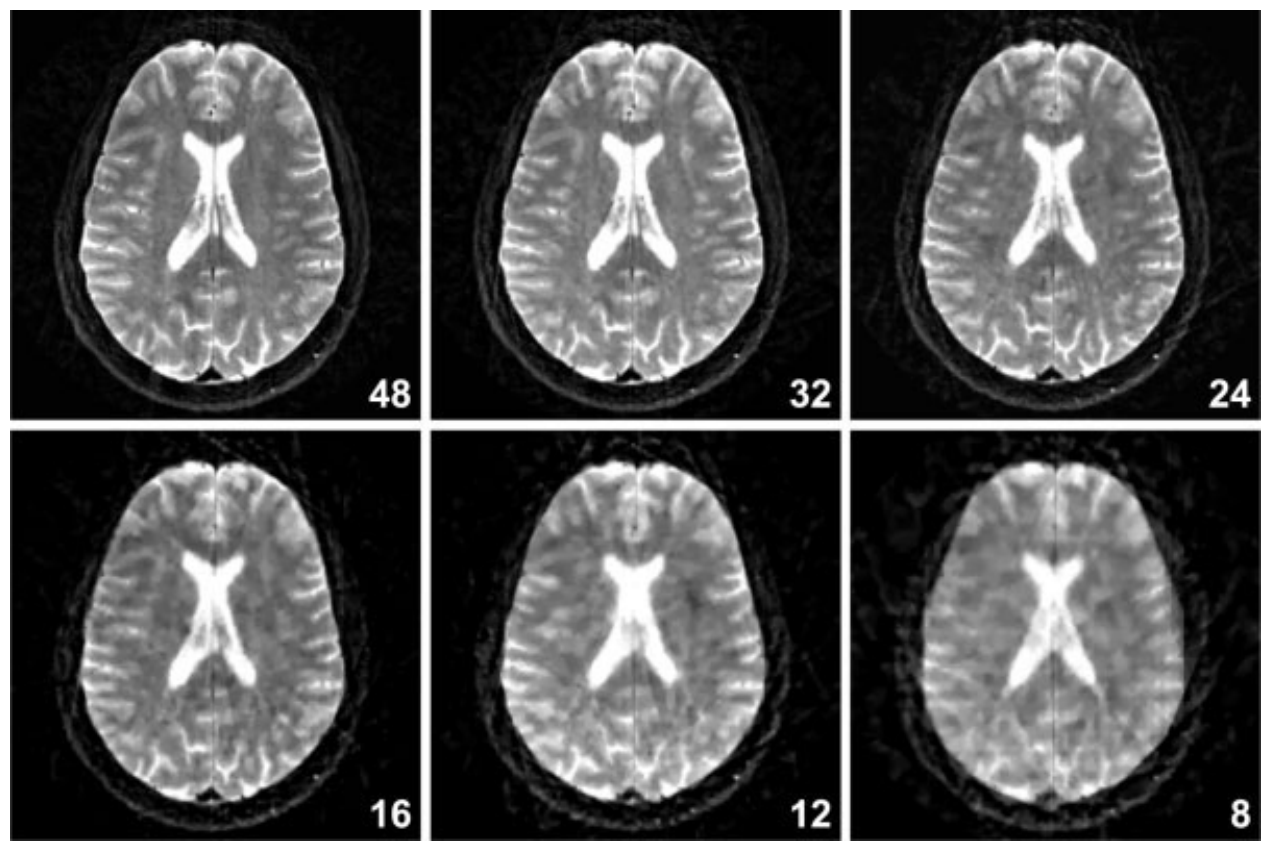
conversely, the total variation cannot be entirely ascribed to the undersampling artifacts. Hence, the more complex the actual object, the less accurate can the information be recovered by restricting the image variation.

Figure 1 illustrates that the width of the streaks from the undersampling increases with decreasing number of spokes. The total variation constraint given by Eq. [12] is based on minimizing the $L_{1}$ norm of the image derivative and therefore especially penalizes intensity oscillations while maintaining sharp edges. Accordingly, the removal of undersampling artifacts by a total variation constraint is most effective if the overlapping streaks create a strongly varying texture as in the case of 64 spokes. In contrast, if the width of the streaks is wide and the object itself has a certain complexity, then after some iterations the total variation of the image becomes dominated by the actual complexity of the object. Further attempts to minimize the total variation then lead to a removal of actual object features. In other words, there is a tradeoff between residual streaking artifacts and the preservation of object details that has to be considered when reconstructing a complex structured object from a low number of spokes. This is demonstrated in Fig. 11 comparing a regridding solution with three iterative reconstructions with an increasing weight on the minimization of the total variation. Because the strongest weight led to a visible removal of object detail, it is recommended to choose the weight of the total variation constraint-given by the coefficient $\lambda_{T V}-$ with respect to the imaging parameters and the object's complexity.

Regardless of this limitation, the proposed reconstruction technique clearly provided visually improved image quality over regridding in all cases tested. Further, it is of course possible to integrate additional or possibly more advanced penalties to support the recovery of unmeasured information in k-space using prior object knowledge. These constraints might be based on multiscale transformations like wavelets or could be motivated by a Bayesian formulation.

A more general problem that arises when reducing the number of spokes (or Fourier lines) is the concomitant decrease of the signal-to-noise ratio (SNR). It also applies to partial Fourier imaging and parallel MRI. It turned out that low SNR poses a more severe limitation for the reconstruction of undersampled radial MRI data sets than the putative loss of resolution. Although the use of the total variation constraint ensures a pronounced denoising while maintaining borders (e.g., compare Fig. 11), it only allows to smooth noise textures but is, of course, incapable to recover object information that is not visible at all due to a low SNR.

\section{Computational Requirements}

Without doubt the iterative reconstruction method is by far more computationally demanding than a regridding or filtered backprojection technique. In fact, only a single evaluation of the functional [7] already doubles the computational load required for regridding, but multiple evaluations during iteration of the conjugate gradient algorithm are required. The duration of a single iteration and the number of iterations needed depends on the degree of undersampling and on the desired reconstruction
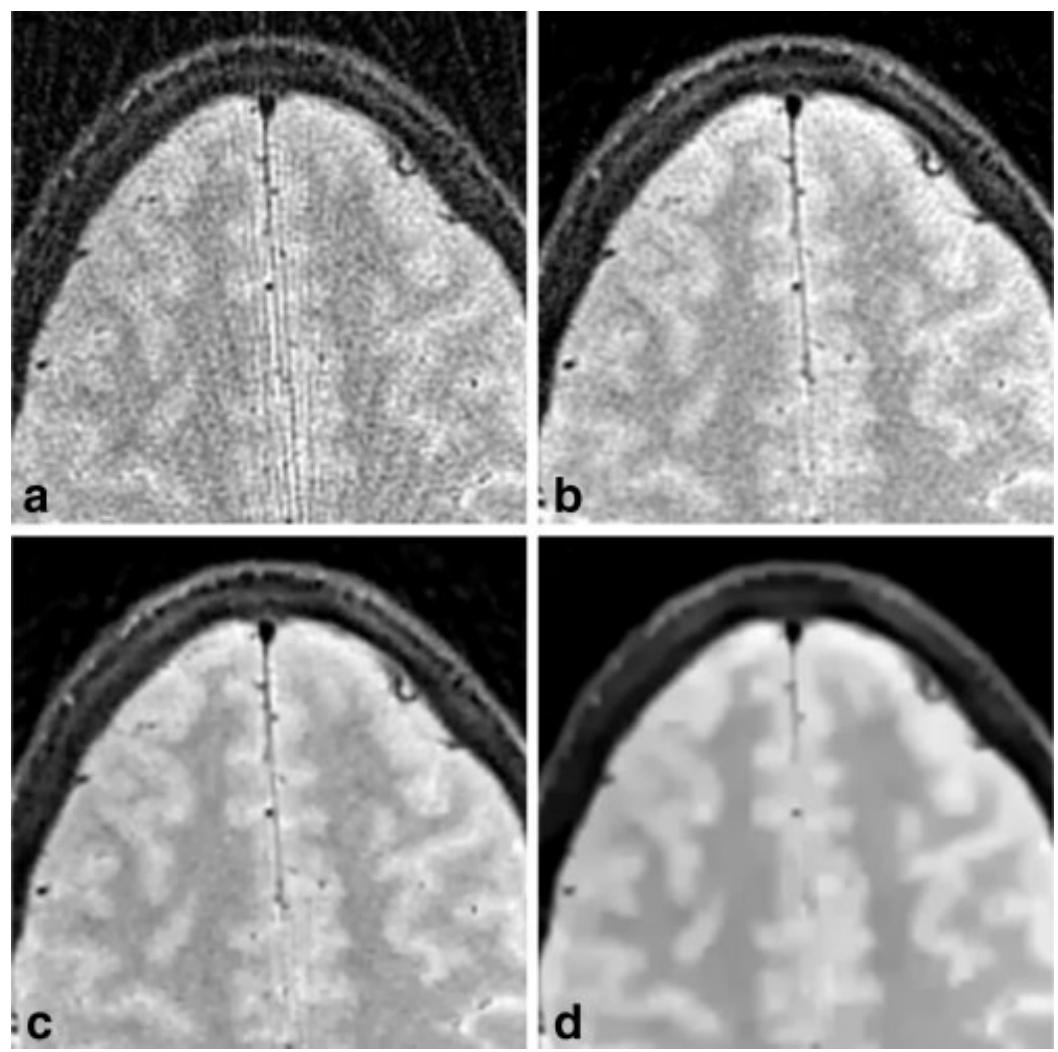

FIG. 11. Radial image reconstructions (human brain, $256 \times 256$ matrix), using experimental data from 48 spokes (256 samples). (a) Regridding and (b-d) the proposed iterative technique with (b) a low weight, (c) an appropriate weight, and (d) an overweight of the total variation constraint. While a proper choice of the total variation penalty yields an efficient denoising without compromising resolution, any overweighting causes a loss of object detail. Parameters as in Fig. 8. 
quality. In general, therefore, it is difficult to give concrete information on reconstruction times.

The present implementation employed a Dell PowerEdge 2900 server with two Intel Xeon $50603.2 \mathrm{GHz}$ dual core processors and $4 \mathrm{~Gb}$ memory for the reconstructions. Images with a base resolution of $256 \times 256$ pixels were rendered using a high number of 30 iterations for each of the 12 channels in the coil estimation step and 120 iterations in the final image reconstruction step. Under these circumstances, the reconstruction of a radial image from 48 spokes took about 520 sec. However, running the reconstruction with only 10 iterations for the coil estimation and 20 iterations for the final reconstruction already resulted in a suitable image quality within only about $120 \mathrm{sec}$. Moreover, the use of only 4 instead of 12 channels further reduced the reconstruction time to $43 \mathrm{sec}$. Finally, there is still potential for optimizing the speed of our implementation. Nevertheless, while these reconstruction times are still too long for a routine clinical setting, steady progress will not take long to render iterative reconstruction techniques more generally suitable for MRI.

\section{Extensions}

An attractive feature of the proposed method is that it can easily be adapted to meet different imaging scenarios by integrating more specific knowledge about the object with use of additional penalty functions. Based on Bayes theorem, basically every kind of a priori knowledge may be incorporated. A tough limitation, though, is that it is necessary to formulate this knowledge such that the problem remains optimizable, which implies at least convex penalty functions.

Furthermore, the system matrix can be extended in order to model the generation of the measured signal in more detail. For example, for a multiecho acquisition, it should be possible to model the signal generation in a timesegmented way. Possibly, this allows to obtain separate density and relaxation maps with improved quality over existing approaches which often mix spokes from different echoes and thereby cause smearing artifacts in areas with strong relaxation. This is because all spokes pass the k-space center and therefore fuse data with inconsistent contrasts.

Another idea would be to include a modelling of offresonance effects, which pose a significant problem for radial gradient-echo MRI. This could be done by using a time-segmented approximation of the local phase evolution based on field maps. For example, the field map could be estimated by shifting the echo time of every other spoke. Subsequently, the reconstruction of separate undersampled images from the odd and even spokes would render coil profiles, a field map, and the final combined image from a single data set.

Although penalizing the total variation is particularly well suited for radial trajectories due to the strongly varying patterns created by radial undersampling, this idea can of course be applied to other trajectories as well. As the technique does not need a density compensation as required for regridding, it allows to reconstruct images from arbitrary trajectories without the need of prior estimates for the sample density using Voronoi diagrams or comparable methods. A second advantage is that the iterative approach reconstructs objects with absolute values that are independent of the amount of data measured. In contrast, for regridding the absolute values of the object usually depend on the total intensity inserted into the raw data matrix.

\section{CONCLUSIONS}

This work presents a technique for the iterative reconstruction of images from undersampled radial MRI acquisitions. The approach is able to handle data from multiple coils and allows to incorporate prior information about the object by introducing suitable penalties. In particular, constraining the total variation of the reconstructions led to an effective reduction of streaking artifacts that normally limit the application of radial undersampling strategies. This enables to obtain images from only a very limited number of spokes with markedly improved quality compared to conventional radial reconstructions. While the current computational speed of the proposed technique is already acceptable for scientific purposes, foreseeable technical progress promises iterative approaches soon to become part of the MRI instrumentarium for more routine applications.

\section{REFERENCES}

1. Lauterbur PD. Image formation by induced local interactions: Examples employing nuclear magnetic resonance. Nature 1973;242:190-191.

2. Mistretta CA, Wieben O, Velikina J, Block W, Perry J, Wu Y, Johnson K, Wu Y. Highly constrained backprojection for time-resolved MRI. Magn Reson Med 2006;55:30-40.

3. Barger AV, Block WF, Toropov Y, Grist TM, Mistretta CA. Time-resolved contrast-enhanced imaging with isotropic resolution and broad coverage using an undersampled 3D projection trajectory. Magn Reson Med 2002;48:297-305

4. Song HK, Dougherty L. k-space weighted image contrast (KWIC) for contrast manipulation in projection reconstruction MRI. Magn Reson Med 2000;44:825-832.

5. Gmitro AF, Kono M, Theilmann RJ, Altbach MI, Li Z, Trouard TP. Radial GRASE: Implementation and applications. Magn Reson Med 2005;53:1363-1371

6. Rahmer J, Boernert P, Groen J, Bos C. Three-dimensional radial ultrashort echo-time imaging with T2 adapted sampling. Magn Reson Med 2006;55:1075-1082.

7. Glover GH, Noll DC. Consistent projection reconstruction (CPR) techniques for MRI. Magn Reson Med 1993;29:345-351.

8. Katoh M, Spuentrup E, Buecker A, Manning WJ, Guenther RW, Botnar RM. MR coronary vessel wall imaging: Comparison between radial and spiral k-space sampling. J Magn Reson Imaging 2006;23:757-762.

9. Trouard TP, Sabharwal Y, Altbach MI, Gmitro AF. Analysis and comparison of motion-correction techniques in diffusion-weighted imaging. J Magn Reson Imaging 1996;6:925-935.

10. Yeh EN, Stuber M, McKenzie CA, Botnar RM, Leiner T, Ohliger MA, Grant AK, Willig-Onwuachi JD, Sodickson DK. Inherently selfcalibrating non-Cartesian parallel imaging. Magn Reson Med 2005;54: 1-8.

11. Liang ZP, Lauterbur PC. Principles of magnetic resonance imaging. IEEE press series on biomedical engineering. New York: IEEE Press; 2000.

12. O'Sullivan JD. A fast sinc function gridding algorithm for Fourier inversion in computer tomography. IEEE T Med Imaging 1985;4:200-207.

13. Jackson J, Meyer CH, Nishimura DG, Macovski A. Selection of a convolution function for Fourier inversion using gridding. IEEE T Med Imaging 1991;10:473-478.

14. Beatty PJ, Nishimura DG, Pauly JM. Rapid gridding reconstruction with a minimal oversampling ratio. IEEE T Med Imaging 2005;24:799-808.

15. Bernstein MA, King FK, Zhou XJ. Handbook of MRI pulse sequences. Burlington. MA: Elsevier Academic Press; 2004.

16. Pruessmann KP, Weiger M, Boernert P, Boesiger P. Advances in sensitivity encoding with arbitrary k-space trajectories. Magn Reson Med 2001;46:638-651. 
17. Hager WW, Zhang H. A survey of nonlinear conjugate gradient methods. Pacific J Optimization 2006;2:35-58.

18. Hager WW, Zhang H. A new conjugate gradient method with guaranteed descent and an efficient line search. SIAM J Optimization 2005;16: 170-192.

19. Delaney AH, Bresler Y. A fast and accurate Fourier algorithm for iterative parallel-beam tomography. IEEE T Med Imaging 1996;5:740-753.

20. Rudin LI, Osher S, Fatemi E. Nonlinear total variation based noise removal algorithms. Physica D 1992;60:259-268.

21. Geman D, Yang C. Nonlinear image recovery with half-quadratic regularization. IEEE T Image Processing 1995;4:932-946.
22. Speier P, Trautwein F. Robust radial imaging with predetermined isotropic gradient delay correction. Proc Int Soc Mag Reson Med 2006; $14: 2379$.

23. Chang TC, He L, Fang T. MR image reconstruction from sparse radial samples using Bregman iteration. Proc Int Soc Mag Reson Med 2006; 14:696.

24. Donoho D. Compressed sensing. IEEE T Information Theory 2006;52: 1289-1306.

25. Candes E, Romberg J, Tao T. Robust uncertainty principles: Exact signal reconstruction from highly incomplete frequency information. IEEE T Information Theory 2006;52:489-509. 